Both these patients reported using neuroleptics intravenously for their sedative properties. Despite this potentially hazardous activity no complications occurred other than mild local phlebitis at the site of injection.

RICHARD DUFFETT and MARTIN LAKER, Royal London Hospital Rotation

\section{No such thing as a free lunch - or a leather-bound desk diary!}

Sir: Dr Azuonye (Psychiatric Bulletin, 1994, 18. 779) provides an interesting glimpse into how consultant psychiatrists view medical representatives. I am surprised he finds that the majority of 'gift-accepting' consultants feel they are not influenced in their choice of drug by these gifts.

We should not forget that medical representatives are employed to sell their products. They are not part of the health service and any gifts or sponsorship they provide are for the purpose of increasing their 'market-share'.

Whether by material gifts or education, drug companies must believe that their representatives influence doctors' prescribing. Let us not kid ourselves otherwise.

Paul RAMchandan,, Newsam Centre, Seacroft Hospital, York Road, Leeds

\section{Community treatment orders}

Sir: We were interested to read Dr Turner's comments on a recent debate at the Royal College of Psychiatrists concerning community treatment orders (CTO) (Psychiatric Bulletin, $1994,18,657-659$ ).

A CTO could prove to be the least restrictive form of treatment for many patients. Its use could be limited to patients who relapse soon after discharge and become potentially dangerous to others because of their noncompliance. It would improve treatment compliance, reduce time spent in hospital and reduce levels of dangerousness (Sensky et al, 1991).

The liberty of the individual should be protected by the Mental Health Act (MHA) 1983. Patients considered at risk on discharge from a section 3 , and previously dangerous to others because of noncompliance, could be assessed by an expanded Mental Health Review Tribunal
(MHRT), a body which already exists to ensure the proper implementation of the MHA. If appropriate, on discharge from section and hospital, restrictions could be imposed regarding compliance with treatment. This would act in a similar way to the existing section 41 restrictions, sometimes added to a section 37 treatment order. We propose the restrictions would be administered by the MHRT.

Secondly, the tribunal could insist on a comprehensive treatment plan for each patient which would include the use of nonpharmacological therapies in addition to medication. Thirdly, the risk of developing adverse effects such as tardive dyskinesia is considerably increased by so-called 'drug holidays' (Glazer et al, 1989) so this cannot be used in argument against a CTO.

The issue of racism within psychiatry must be discussed but is not a valid argument against at CTO. To do so prevents progress but not racism. Once again the tribunal would be expected to prevent potential abuses.

It is disturbing to hear the suggestion that compulsory treatment in the community would be "community care on the cheap". Given that more research is required to enable the costing of community care it would be facetious to argue that proposing a CTO is driven by economics and not a wish to see more responsive mental health legislation.

As doctors we are more interested in appropriate medical treatment for our patients and less in the politics of detention. While previously these two provisions were complementary, the move to the community means this is no longer so and we must now insist that we are only prepared to take responsibility for the treatment of dangerous non-compliant out-patients if we have the backing of statutes to enforce it. Furthermore, if the state denies us this then it should not attempt to apportion blame with the use of supervision registers, which are in any case contrary to the tenets of patient confidentiality.

Glazer, W. M., Bowers, M. B. Jr., Charney, D. S. \& Heninger, G. R. (1989). The effect of neuroleptic discontinuation on psychopathology, involuntary movements and biochemical measures in patients with persistent tardive dyskinesia. Biological Psychiatry, 26. 224-233.

SENSKY, T., Hughes, T. \& HIRSCH, S. (1991) Compulsory psychiatric treatment in the community. British Joumal of Psychiatry. 168, 792-799.

MARTIN Lock, Three Bridges Regional Secure Unit, Ealing Hospital, Southall UBI 3EU; and 
DigBY QUESTED, Senior Registrar, Horton Hospital, Long Grove Road, Epsom KT19 8PZ

\section{The use of clozapine}

Sir: In the audit article on the use of clozapine in South Manchester (Seabourne \& Thomas, Psychiatric Bulletin, 1994, 18, 618-619) 25 patients were given clozapine between 1990 and 1992 and, at the end of the trial, only ten subjects were still receiving the drug, the majority having been discontinued because of side-effects.

I am interested in why patients who have started clozapine are then terminated. Neutropenia apparently accounted for only one patient and the other side-effects noted were hypersalivation, sedation, grand mal fits, myoclonic jerks, vomiting, neuroleptic malignant syndrome, acute confusional state with cognitive impairment, slurred speech, benign hyperthermia, dry mouth, weight gain, constipation, diarrhoea, hypotension, and urinary incontinence. The clue may lie in the mean daily dose of clozapine; around $445 \mathrm{mg}$ per day for responders, $633 \mathrm{mg}$ per day for non-responders and $356 \mathrm{mg}$ per day for the side-effect patients. This dosage is markedly above the average UK dosage of around $300 \mathrm{mg}$ per day and our own dosage which is nearer to $200 \mathrm{mg}$ per day.

With the experience of around 70 patients in the community on clozapine I would say that these patients were probably receiving clozapine at too high a dosage. Apart from the case of neuroleptic malignant syndrome, and of neutropenia, these side-effects are dose related. If the clozapine is combined with an oral conventional anti-psychotic then any breakthrough psychotic symptoms can be usually managed. In this way most of the side-effects would have markedly reduced and, with regards to epileptic and myoclonic sideeffects that were not reduced, better control could be achieved with the addition of an anticonvulsant, e.g. sodium valproate.

With these modifications we might have seen an improvement in the high final discontinuation rate of $32 \%$. Another benefit of lower dosage is that patients are less likely to non-comply as they perceive an enhanced life quality.

The authors mention that they are interested in maximising the response to clozapine so as to persuade the purchasers to spend more money on the drug. A spin-off from using combined clozapine therapy is that the cost is less and you can get more well patients for your money.

MichAel LAUNER, Burnley Healthcare NHS Trust, Burnley General Hospital, Burnley $B B 102 P Q$

Sir: We agree with Dr Launer's observation that the doses of clozapine administered to patients were high. However, King \& Mills (1993) reported doses of $438 \mathrm{mg}$ a day in females and $488 \mathrm{mg}$ a day in males; and Meltzer (1992) recommended a target dose of $450 \mathrm{mg}$ a day given as monotherapy for six months. If the response was inadequate after this time it was suggested that doses up to $900 \mathrm{mg}$ a day should be tried.

Our audit showed that patients experiencing side-effects were on lower doses (356 mg) than those who responded $(445 \mathrm{mg})$, who in turn were on lower doses than non-responders $(633 \mathrm{mg})$. It is possible that lower doses may have been better tolerated and fewer patients would have been withdrawn from treatment. It is becoming clear that some patients can be maintained on low doses with two of our patients receiving $75 \mathrm{mgs}$ and $100 \mathrm{mgs}$ a day.

We dispute the logic of combining clozapine with an oral conventional antipsychotic as a way of reducing side effects. The BNF states that "prescribing of more than one antipsychotic at the same time is not recommended; it may constitute a hazard and there is not significant evidence that side effects are minimised". The Clozaril Patient Monitoring Service (CPMS) report that there are no absolute contraindications to combining other neuroleptics with clozapine. However, combinations should be used with care especially in early clozapine therapy as this may prolong neutropenia, particularly if depot medication is used. In our experience monotherapy is preferable, although sodium valproate was used in the two subjects who developed seizures.

We are also unaware of any cost-benefit analyses which support Dr Launer's final statement that combined clozapine therapy costs less and you can get more well patients for your money. There are cost benefit analyses which demonstrate that clozapine when compared with conventional neuroleptics significantly improves social functioning, quality of life and reduced the need for inpatient admission in the second and following 